\title{
Detecting Stress Granules in Drosophila neurons
}

Fabienne De Graeve ${ }^{1, \#}$, Nadia Formicola ${ }^{1, \#}$, Kavya Pushpalatha ${ }^{1, \#}$, Akira Nakamura $^{2}$, Eric Debreuve ${ }^{3}$, Xavier Descombes ${ }^{4}$ and Florence Besse ${ }^{1, *}$

1- Université Côte d'Azur, CNRS, Inserm, Institut de Biologie Valrose, Nice, France

2- Department of Germline Development, Institute of Molecular Embryology and Genetics, and Graduate School of Pharmaceutical Sciences, Kumamoto University, Kumamoto, Japan

3- Université Côte d'Azur, CNRS, Inria, Laboratoire I3S, France

4- Université Côte d'Azur, Inria, CNRS, Laboratoire I3S, France

\# contributed equally to the work

* corresponding author: besse@unice.fr 


\begin{abstract}
Stress granules (SGs) are cytoplasmic ribonucleoprotein condensates that dynamically and reversibly assemble in response to acute or chronic stress. They are thought to contribute to the adaptive stress response by storing translationally inactive mRNAs as well as signaling molecules. Recent work has shown that SG composition and properties depend on both stress and cell types, and that neurons exhibit a complex SG proteome and a strong vulnerability to mutations in SG proteins. Drosophila has emerged as a powerful genetically tractable organism where to study the physiological regulation and functions of SGs in normal and pathological contexts. In this chapter, we describe a protocol enabling quantitative analysis of SG properties in both larval and adult Drosophila CNS samples. In this protocol, fluorescently-tagged SGs are induced upon acute ex vivo stress or chronic in vivo stress, imaged at high-resolution via confocal microscopy and detected automatically, using a dedicated software.
\end{abstract}

Keywords: central nervous system, confocal imaging, fluorescent stress granule proteins, automated detection, Drosophila melanogaster 


\section{Introduction}

Cellular stress induces a translational shutdown within minutes, characterized by inhibition of translation initiation and polysome disassembly. Cytoplasmic release of translationally inactive mRNAs in turn triggers the assembly of hundreds of nanometer-sized membraneless compartments enriched in stalled housekeeping transcripts and associated proteins, and referred to as stress granules (SGs) (Protter and Parker 2016; Riggs et al. 2020). These higher order ribonucleoprotein (RNP) assemblies behave as dynamic condensates: they form through the self-association of their constituents into dense networks of transient RNA-RNA, RNA-protein and protein-protein interactions and get actively disassembled upon stress release (Mittag and Parker 2018; Protter et al. 2018; Hofmann et al. 2020). The rapid and reversible mode of SG assembly is thought to play important roles in the adaptive stress response, first by promoting translational reprogramming through transient sequestration of unnecessary RNAs, and second by rewiring cellular pathways through recruitment of signaling molecules (Buchan and Parker 2009; Kedersha et al. 2013). Consistent with the functional importance of SG dynamics, extensive links have recently been established between alterations of SG material properties and neurodegenerative diseases ( $\mathrm{Li}$ et al. 2013; Formicola et al. 2019; Wolozin and Ivanov 2019). Abnormally stable inclusions enriched in SG components, for example, have been observed in pathological contexts and defined as a characteristic signature of amyotrophic lateral sclerosis (ALS) or frontotemporal dementia (FTD) patient samples (Neumann et al. 2006; Wolozin and Ivanov 2019). Furthermore, mutations in an increasing number of SG components, including the RNA binding proteins TDP-43, FUS or TIA1, have been causally linked to disease progression and shown to promote the transition of RNP assemblies into irreversible solid-like condensates (Sreedharan et al. 2008; Vance et al. 2009; Li et al. 2013; Patel et al. 2015; Mackenzie et al. 2017; Wolozin and Ivanov 2019). As revealed by a recent systematic study, the pathological entities formed upon expression of ALS mutant proteins also have a composition distinct from their dynamic and reversible counterparts (Markmiller et al. 2018), highlighting their capacity to recruit, and potentially titrate molecules involved in RNA homeostasis. More work is now required to decipher if and how pathological SGs induce toxicity in neuronal cells, which, as long-lived non-dividing cells, appear to 
be particularly vulnerable to the chronic stress induced by mutant SG proteins (Li et al. 2013). Importantly, proteomic studies have uncovered that variations in the composition of SGs are also observed in normal contexts in function of cell types and nature of the stress (Markmiller et al. 2018; Advani and Ivanov 2020). While a core set of obligatory components, including factors essential for SG nucleation, has been found in the different cell types analyzed, a significant fraction of the SG proteome was shown to be recruited exclusively in certain cell types, particularly in neurons (Markmiller et al. 2018; Advani and Ivanov 2020). Together, these studies have uncovered an unexpected diversity and highlighted the limits of working with standard immortalized cell lines. They have raised the need to develop alternative biological models in which SG regulation and function can be studied under physiological conditions, in differentiated tissues.

Drosophila represents an excellent model organism in which advanced genetics can be combined with high-resolution imaging to unravel the mechanisms underlying SG assembly, as well as SG function in adaptation to environmental stress or disease-associated chronic stress. Fly orthologs of mammalian SG components, indeed, were shown to accumulate within cytoplasmic condensates in response to different acute stresses including oxidative stress, Endoplasmic Reticulum (ER) stress or hypoxia (Farny et al. 2009; van der Laan et al. 2012; Gareau et al. 2013; Jevtov et al. 2015; Bakthavachalu et al. 2018; Buddika et al. 2020). Furthermore, various Drosophila ALS models have been developed, in which SG proteins with disease-causing mutations are chronically expressed in the nervous system (Chen et al. 2011; Estes et al. 2011; Lanson et al. 2011; McGurk et al. 2015). These models were shown to recapitulate many aspects of the disease, among which cytoplasmic accumulation of pathological SG-like assemblies (Chen et al. 2011; Estes et al. 2011; Alami et al. 2014; De Graeve et al. 2019). Here, we describe a protocol that enables induction of Stress Granules in the nervous system of Drosophila, either chronically in response to in vivo expression of pathological SG proteins, or acutely upon treatment of explants with stress inducers (e.g. arsenite). This protocol includes the procedure to perform high-resolution confocal imaging of fluorescently-tagged SG markers and to accurately and automatically detect SGs using the Obj.MPP software (De Graeve et al. 2019). The described method is compatible with analysis of both larval and adult central nervous 
system (CNS) samples, and is particularly adapted to the quantitative analysis of SG properties in complex tissues.

\section{Materials}

\section{1- Fly lines for expression of fluorescent SG proteins}

1. Gal4 and UAS transgenic flies for conditional ectopic expression of fluorescent pathological SG proteins in the nervous system (e.g. OK371-Gal4 and UAS-TDP-43 fly lines; see Table 1).

2. Knock-in lines expressing fluorescent SG proteins from the endogenous locus (e.g. GFP-Rasputin (Rin; the fly ortholog of G3BP); see Table 1).

\section{2- Arsenite treatment}

1- Chambered slide, four wells (see Note 1).

2- Preparation of arsenite stock solution: weight sodium (meta)arsenite powder and dissolve in freshly prepared HL3 (see 2.3.2) to obtain a $40 \mathrm{mM}$ stock solution that can be stored at room temperature (see Note 2). Alternatively, purchase commercially available aqueous solution.

\section{3- Dissection and fixation of Drosophila CNS samples}

\subsection{1- Dissection and fixation of larval CNS}

1. A pair of dissection forceps.

2. $60 \mathrm{~mm}$ dissection petri dish.

3. 1X Phosphate Buffer Saline (PBS) (see Note 3).

4. Fixing solution: 4\% formaldehyde in $1 \mathrm{X}$ PBS (see Note 3).

\subsection{2- Dissection and fixation of adult brains}

1. A pair of dissection forceps.

2. Minutien pins.

3. $60 \mathrm{~mm}$ dissection petri dish covered with $2 \%$ agarose. 
4. HL3 buffer $(70 \mathrm{mM} \mathrm{NaCl}, 5 \mathrm{mM} \mathrm{KCl}, 4 \mathrm{mM} \mathrm{MgCl} 2,5 \mathrm{mM}$ trealose, $115 \mathrm{mM}$ sucrose, $5 \mathrm{mM}$ HEPES, 10 mM NaHCO 3 , pH 7.20-7.25) (see Note 4).

5. Fixing solution: 4\% formaldehyde in HL3.

6. Wash buffer: PBS; $0.5 \%$ Triton-X.

\section{4- Mounting of Drosophila CNS samples}

1. Antifade mounting medium with DAPI (Vectashield).

2. 10-well glass slides (black teflon coating).

3. $1.5,24 X 60 \mathrm{~mm}$ coverslips.

\section{5- Image acquisition}

1. Scanning confocal microscope with highly sensitive detectors.

2. 63X 1.4 NA oil objective.

3. Immersion oil.

\section{6- Image analysis}

1. ImageJ/FIJI (https://imagej.net/Fiji).

2. Obj.MPP software (De Graeve et al. 2019) (https://gitlab.inria.fr/edebreuv/Obj.MPP).

\section{Methods}

In this protocol, stress can either be applied endogenously (3.1.1) or exogenously (3.1.2) (Fig. 1.). Note that dissection of Drosophila nervous system (3.2) is performed after stress induction in case of endogenous stress and before stress in case of exogenous stress (Fig. 1).

\section{1- Induction of stress}

\subsection{1- Ectopic in vivo expression of pathological proteins}


1. Cross transgenic flies expressing a fluorescently-tagged pathological SG protein under UAS control (e.g. UAS-Venus-TDP-43 M337V; see Table 1) with flies expressing a neuronal Gal4 driver (e.g. motorneuron OK371-Gal4; see Table 1) (Fig. 1, upper left).

2. Maintain the flies at $25^{\circ} \mathrm{C}$ and transfer them in a new vial every 3-4 days (see Note 5).

\subsection{2- Ex vivo treatment with arsenite}

1- Freshly prepare the working arsenite solution $(0.4 \mathrm{mM})$ by diluting the stock solution into HL3 (see

\section{Note 2).}

2- Transfer dissected samples in a multi-well chambered slide (Fig. 1, upper right). At least 15 samples should be treated per condition.

3- Incubate in $500 \mu \mathrm{L}$ of $\mathrm{HL} 3$ or arsenite solution for one hour at $25^{\circ} \mathrm{C}$, covered from light.

\section{2- Dissection of Drosophila CNS samples}

\subsection{1-Dissection of larval CNS}

1. Collect wandering third instar larvae expressing normal or pathological fluorescent SG proteins.

2. Cut the larvae in two using a pair of forceps.

3. Turn the anterior half of larvae inside out, remove the fat tissue while keeping the CNS attached to the cuticle. Collect samples using a $1 \mathrm{~mL}$ pipet tip.

\subsection{2-Dissection of adult brains}

1. Collect 7-10 day-old flies expressing normal or pathological Stress Granule proteins and anesthetize them with $\mathrm{CO} 2$.

2. Dissect brains in HL3 buffer, as described in (Williamson and Hiesinger 2010; Tito et al. 2016). Briefly, immobilize the flies ventral side up by pinning them in a dissecting dish filled with HL3. Pull the proboscis upwards with one forceps and insert the tips of the other forceps underneath, in a closed position. Slowly open the forceps so as to tear apart the head cuticle. Carefully remove the cuticle and the retina, without damaging the underlying optic lobes and central brain. 
3. Complete the dissection by thoroughly removing the air sacs (see Note 6).

4. Separate the brains from the rest of the body. The dissected brains can be collected using a glass pipette or a filtered tip (see Note 7).

\section{3- Fixation of Drosophila CNS samples}

This step comes right after dissection in case of endogenous stress induction or after treatment of brain explants in case of ex vivo arsenite treatment.

\section{$\underline{\text { 3.3.1- Fixation of larval CNS }}$}

1. Transfer the larval samples into microtubes.

2. Add $500 \mu \mathrm{L}$ of fixing solution and gently rock the samples for 20 minutes at room temperature (RT).

3. Replace the fixing solution with $1 \mathrm{~mL}$ of $1 \mathrm{X}$ PBS and gently rock the samples for 30 minutes at $\mathrm{RT}$.

4. Repeat step 3 twice.

5. Remove 1X PBS and add a drop of antifade mounting medium supplemented with DAPI.

6. Keep at $4^{\circ} \mathrm{C}$ for a minimum of 2 hours (preferentially overnight).

\subsection{2- Fixation of adult brains}

1. Transfer the brains into microtubes.

2. Add $300 \mu \mathrm{L}$ of fixing solution and gently rock the samples for 25 minutes at room temperature (RT).

3. Remove the fixing solution, replace with $800 \mu \mathrm{L}$ of wash buffer and gently rock the samples for 30 minutes at RT.

4. Repeat step 3 twice.

5. Remove the wash buffer and add a drop of antifade mounting medium supplemented with DAPI.

6. Keep at $4^{\circ} \mathrm{C}$ for a minimum of 2 hours (preferentially overnight).

\section{4- Mounting of Drosophila CNS samples}




\subsection{1- Mounting of larval CNS}

1. Transfer the samples onto a dissection dish using a $1 \mathrm{~mL}$ pipet tip and dissect the samples further by detaching the CNS from the cuticle and removing eye-antenna imaginal discs. Recover the brain lobes and ventral nerve cord.

2. Transfer the clean CNS to a multi-well slide ( 5 CNS per well) (see Notes 8,9$)$.

3. Orient the larval CNS with forceps, such that the dorsal side of the ventral cord is up.

4. Carefully place a $24 \times 60 \mathrm{~mm}$ coverslip on top of the slide and seal the coverslip with clear nail varnish.

\subsection{2-Mounting of adult brains}

1. Transfer the brains to a multi-well slide ( $~ 5$ CNS per well) (see Notes 8,9 ).

2. Orient the brains with forceps, such that their dorsal side is up.

3. Carefully place a $24 \times 60 \mathrm{~mm}$ coverslip on top of the slide and seal the coverslip with clear nail varnish.

\section{5- Imaging of Drosophila CNS samples}

1. Acquire images from larval CNS or adult brains with a confocal microscope equipped with highsensitivity detectors, and appropriate laser lines (see Note 10).

2. Image with optimal resolution (see Note 11), using a 63X 1.4 NA oil objective.

3. SGs appear as discrete, bright cytoplasmic foci with a typical diameter of hundreds nanometers (Fig. 2B and D).

\section{6- Image analysis: detection of Stress Granules}

1. Using ImageJ/Fiji, select single optical sections and crop to generate stereotypic regions of interest. Save images in .tif format, in a single dedicated folder.

2. Launch the Obj.MPP software (see Note 12).

3. Select image(s) to be analyzed in the first tab of the GUI. 
4. Select the detection parameters in the second tab of the GUI (Fig. 3). These parameters include object types and expected size range (see Note 13), as well as object radiometric properties (defined by the quality function; see Note 14).

5. Set the number of iterations in the third tab of the GUI (see Note 15).

6. Select output files (see Note 16) and output path in the fourth tab of the GUI.

\section{Notes}

1. The multi-well chambered slides can be rigorously washed with ethanol $80 \%$ and re-used up to three times.

2. Sodium Arsenite is a hazardous substance classified as carninogen, mutagen and teratogen; it should be handled safely, under a chemical hood. When solubilized, sodium arsenite should be stored as sealed aliquots covered from light to avoid oxidation.

3. Prefer HL3 in case long incubations are required (if applying ex vivo stress).

4. HL3 buffer contains sugars (sucrose and trehalose) and can easily get contaminated. Store at $4^{\circ} \mathrm{C}$ in aliquots sealed with parafilm. Opened aliquots should not be kept for more than 2 months.

5. Temperature should be adapted so as to permit high expression level while preventing toxicity.

6. If air sacs are not removed, brains will float, making it difficult to not pipet them away.

7. Pre-wetting the pipette tip or the glass pipette with HL3 prevents the brains from sticking to the plastic/glass wall.

8. Do not place samples in wells close to the edge of the slide; they will not be accessible on regular microscope stages.

9. Transfer samples in a drop of mounting medium only, as excess medium can make brains float over the edge of the wells.

10. We used a confocal microscope equipped with ultrasensitive detectors (Zeiss LSM 880 with gallium arsenide phosphide (GaAsp) detectors). 
11. Imaging with a xy pixel size of less than $80 \mathrm{~nm}$ is recommended. We imaged larval CNS with a xy pixel size of $74 \mathrm{~nm}$ (regular confocal microscopy), and adult brains with a xy pixel size of $45 \mathrm{~nm}$ (Airy scan confocal microscopy).

12. Obj.MPP can be used either through the graphical user interface (GUI) or through a terminal console (Command-Line Interface (CLI)). More parameters can be adjusted when using the latter mode (see https://edebreuv.gitlabpages.inria.fr/Obj.MPP/).

13. Object types and their corresponding parameters (notably size and orientation) are described under: https://edebreuv.gitlabpages.inria.fr/Obj.MPP/contents/users/object-types.html. Superquadrics are typically recommended for detection of objects with potentially complex shapes such as Stress Granules. We used the following parameter ranges for detection of Stress Granules from larval CNS and adult brains: semi_minor_axis_range $=(2,4,0.25)$, major_minor_ratio_range $=(1.0,1.5,0.025)$, exponent_range $=(1.5,2.5,0.1)$, angle_degree_range $=(0.0,179.9,5.0)$ (see Note 14 about mpp_quality_chooser.py).

14. Available quality functions and associated signal transformations are described under: https://edebreuv.gitlabpages.inria.fr/Obj.MPP/contents/users/quality-measures.html. Note that mpp_quality_chooser.py (https://edebreuv.gitlabpages.inria.fr/Obj.MPP/contents/users/mpp-qualitychooser.html) can be used to identify the best quality function and parameter ranges to detect objects of interest. We used the bright-on-dark gradient quality function with a min_quality of 1.5.

15. The number of iterations and the number of births per iteration should be set so that best objects are all reproducibly retained at the end of the process. We used 1.500 iterations with 50 births per iteration.

16. Different outputs can be selected in the last tab of the GUI, including: CSV files containing the characteristics of the detected granules (geometrical parameters, intensity), raw images with granule contours highlighted, or masks of the detected granules, each having its own label (Fig. 4).

\section{Acknowledgements}


Development of this protocol was supported by the CNRS, as well as grants from the ANR (ANR-15CE12-0016 and ANR-20-CE16-) and the Fondation pour la Recherche Médicale (Equipe FRM; grant \#DEQ20180339161) to F.B. Part of this work was also supported by the Joint Usage/Research Center for Developmental Medicine, IMEG, Kumamoto University. N.F. and K.P. were supported by fellowships from the LABEX SIGNALIFE program (\#ANR - 11 - LABX - 0028 - 01). K.P. was in addition supported by a one year- La Ligue contre le cancer fellowship. We thank the iBV PRISM Imaging facility for use of their microscopes and support (especially B. Monterroso), and L. Palin for excellent technical assistance.

\section{References}

Advani VM, Ivanov P. 2020. Stress granule subtypes: an emerging link to neurodegeneration. Cellular and molecular life sciences: CMLS 77: 4827-4845.

Alami NH, Smith RB, Carrasco MA, Williams LA, Winborn CS, Han SSW, Kiskinis E, Winborn B, Freibaum BD, Kanagaraj A et al. 2014. Axonal transport of TDP-43 mRNA granules is impaired by ALS-causing mutations. Neuron 81: 536-543.

Bakthavachalu B, Huelsmeier J, Sudhakaran IP, Hillebrand J, Singh A, Petrauskas A, Thiagarajan D, Sankaranarayanan M, Mizoue L, Anderson EN et al. 2018. RNP-Granule Assembly via Ataxin-2 Disordered Domains Is Required for Long-Term Memory and Neurodegeneration. Neuron 98: 754-766.e754.

Buchan JR, Parker R. 2009. Eukaryotic stress granules: the ins and outs of translation. Molecular cell 36: $932-941$.

Buddika K, Ariyapala IS, Hazuga MA, Riffert D, Sokol NS. 2020. Canonical nucleators are dispensable for stress granule assembly in Drosophila intestinal progenitors. Journal of cell science 133.

Chen Y, Yang M, Deng J, Chen X, Ye Y, Zhu L, Liu J, Ye H, Shen Y, Li Y et al. 2011. Expression of human FUS protein in Drosophila leads to progressive neurodegeneration. Protein \& cell 2: 477-486.

De Graeve F, Debreuve E, Rahmoun S, Ecsedi S, Bahri A, Hubstenberger A, Descombes X, Besse F. 2019. Detecting and quantifying stress granules in tissues of multicellular organisms with the Obj.MPP analysis tool. Traffic (Copenhagen, Denmark) 20: 697-711. 
Estes PS, Boehringer A, Zwick R, Tang JE, Grigsby B, Zarnescu DC. 2011. Wild-type and A315T mutant TDP-43 exert differential neurotoxicity in a Drosophila model of ALS. Human molecular genetics 20: 2308-2321.

Farny NG, Kedersha NL, Silver PA. 2009. Metazoan stress granule assembly is mediated by PeIF2alpha-dependent and -independent mechanisms. RNA (New York, NY) 15: 1814-1821.

Formicola N, Vijayakumar J, Besse F. 2019. Neuronal ribonucleoprotein granules: Dynamic sensors of localized signals. Traffic (Copenhagen, Denmark) 20: 639-649.

Gareau C, Houssin E, Martel D, Coudert L, Mellaoui S, Huot ME, Laprise P, Mazroui R. 2013. Characterization of fragile $\mathrm{X}$ mental retardation protein recruitment and dynamics in Drosophila stress granules. PloS one 8: e55342.

Hofmann S, Kedersha N, Anderson P, Ivanov P. 2020. Molecular mechanisms of stress granule assembly and disassembly. Biochimica et biophysica acta Molecular cell research 1868: 118876.

Jevtov I, Zacharogianni M, van Oorschot MM, van Zadelhoff G, Aguilera-Gomez A, Vuillez I, Braakman I, Hafen E, Stocker H, Rabouille C. 2015. TORC2 mediates the heat stress response in Drosophila by promoting the formation of stress granules. Journal of cell science 128: 2497-2508.

Kedersha N, Ivanov P, Anderson P. 2013. Stress granules and cell signaling: more than just a passing phase? Trends in biochemical sciences 38: 494-506.

Lanson NA, Jr., Maltare A, King H, Smith R, Kim JH, Taylor JP, Lloyd TE, Pandey UB. 2011. A Drosophila model of FUS-related neurodegeneration reveals genetic interaction between FUS and TDP-43. Human molecular genetics 20: 2510-2523.

Li YR, King OD, Shorter J, Gitler AD. 2013. Stress granules as crucibles of ALS pathogenesis. The Journal of cell biology 201: 361-372.

Mackenzie IR, Nicholson AM, Sarkar M, Messing J, Purice MD, Pottier C, Annu K, Baker M, Perkerson RB, Kurti A et al. 2017. TIA1 Mutations in Amyotrophic Lateral Sclerosis and Frontotemporal Dementia Promote Phase Separation and Alter Stress Granule Dynamics. Neuron 95: 808-816.e809.

Markmiller S, Soltanieh S, Server KL, Mak R, Jin W, Fang MY, Luo EC, Krach F, Yang D, Sen A et al. 2018. Context-Dependent and Disease-Specific Diversity in Protein Interactions within Stress Granules. Cell 172: 590-604.e513.

McGurk L, Berson A, Bonini NM. 2015. Drosophila as an In Vivo Model for Human Neurodegenerative Disease. Genetics 201: 377-402.

Mittag T, Parker R. 2018. Multiple Modes of Protein-Protein Interactions Promote RNP Granule Assembly. J Mol Biol 430: 4636-4649. 
Neumann M, Sampathu DM, Kwong LK, Truax AC, Micsenyi MC, Chou TT, Bruce J, Schuck T, Grossman M, Clark CM et al. 2006. Ubiquitinated TDP-43 in frontotemporal lobar degeneration and amyotrophic lateral sclerosis. Science 314: 130-133.

Patel A, Lee HO, Jawerth L, Maharana S, Jahnel M, Hein MY, Stoynov S, Mahamid J, Saha S, Franzmann TM et al. 2015. A Liquid-to-Solid Phase Transition of the ALS Protein FUS Accelerated by Disease Mutation. Cell 162: 1066-1077.

Protter DSW, Parker R. 2016. Principles and Properties of Stress Granules. Trends in cell biology 26: 668-679.

Protter DSW, Rao BS, Van Treeck B, Lin Y, Mizoue L, Rosen MK, Parker R. 2018. Intrinsically Disordered Regions Can Contribute Promiscuous Interactions to RNP Granule Assembly. Cell Rep 22: 1401-1412.

Riggs CL, Kedersha N, Ivanov P, Anderson P. 2020. Mammalian stress granules and P bodies at a glance. Journal of cell science $\mathbf{1 3 3}$.

Sreedharan J, Blair IP, Tripathi VB, Hu X, Vance C, Rogelj B, Ackerley S, Durnall JC, Williams KL, Buratti E et al. 2008. TDP-43 mutations in familial and sporadic amyotrophic lateral sclerosis. Science 319: 1668-1672.

Tito AJ, Cheema S, Jiang M, Zhang S. 2016. A Simple One-step Dissection Protocol for Whole-mount Preparation of Adult Drosophila Brains. Journal of visualized experiments : JoVE.

van der Laan AM, van Gemert AM, Dirks RW, Noordermeer JN, Fradkin LG, Tanke HJ, Jost CR. 2012. mRNA cycles through hypoxia-induced stress granules in live Drosophila embryonic muscles. The International journal of developmental biology 56: 701-709.

Vance C, Rogelj B, Hortobagyi T, De Vos KJ, Nishimura AL, Sreedharan J, Hu X, Smith B, Ruddy D, Wright $\mathrm{P}$ et al. 2009. Mutations in FUS, an RNA processing protein, cause familial amyotrophic lateral sclerosis type 6 . Science 323: 1208-1211.

Williamson WR, Hiesinger PR. 2010. Preparation of developing and adult Drosophila brains and retinae for live imaging. Journal of visualized experiments : JoVE.

Wolozin B, Ivanov P. 2019. Stress granules and neurodegeneration. Nature reviews Neuroscience 20: 649-666. 


\section{Figure legends}

\section{Figure 1. Method workflow.}

Induction of stress in Drosophila nervous system was performed either endogenously (left, green panel) or exogenously (right, orange panel). For the endogenous strategy, expression of fluorescent pathological stress granule proteins is induced chronically in vivo using the Gal4/UAS system. Larval or adult progenies expressing the mutant fluorescent SG markers in neurons are dissected and their CNS/brain collected. Ex vivo stress induction is achieved through acute arsenite treatment of larval $\mathrm{CNS} /$ brain explants dissected from larvae or adults expressing endogenous fluorescent SG proteins. In both procedures, stress induction and dissection are followed by sample fixation, mounting and confocal imaging (lower panel). Automated detection of SGs is performed via the Obj.MPP software.

\section{Figure 2. Imaging of SGs in larval and adult Drosophila CNS.}

(A) Schematic representation of a third instar larva ventral nerve cord with the position of OK371Gal4-expressing motorneuron soma highlighted in green. (B) Confocal image (MIP projection) of the ventral nerve cord of a third instar larva chronically expressing Venus::TDP-43 M337V in motorneurons (OK371-Gal4/+; UAS-Venus::TDP43 M337V/+). Scale bar: . Note the presence of pathological aggregates in motorneuron cytoplasm (arrows). (C) Schematic representation of an adult brain expressing Rasputin (Rin)-GFP proteins from the endogenous locus. (D) Low magnification view of Rin-GFP distribution in the absence of stress. Rin-GFP protein localizes broadly in the Drosophila brain; it is present in the cell bodies of Mushroom Body neurons (MB). (E) In the absence of stress (left), Rin-GFP proteins exhibit diffuse cytoplasmic distribution in MB neurons. Upon arsenite treatment (right), Rin-GFP localizes to SGs. Scale bar....

\section{Figure 3. Obj.MPP graphical user interface (GUI).}

The second tab of the Obj.MPP GUI is shown, in which detection parameters including type and size ranges of objects, as well as threshold for the quality function, must be selected. Parameter values adapted to the detection of SGs in larval motorneurons are displayed.

Figure 4. Obj.MPP-based detection of SGs on confocal images of Drosophila larval and adult CNS.

(A) Automated detection of Venus::TDP-43 M337V-positive SG granules in motorneurons of third instar larva. Left : single confocal section showing SGs accumulating in cell bodies of a motorneuron. Middle : overlay of the raw confocal image and the Obj.MPP detection. Right : Mask of the detected objects. Scale bar : $3 \mu \mathrm{m}$. The plain and dotted lines delimit the contour of the cell and nucleus, respectively. (B) Automated detection of Rin-GFP-positive SGs induced in response to Arsenite. Left : single confocal section showing SGs accumulating in cell bodies of MB neurons. Middle: overlay of 
the raw confocal image and the Obj.MPP detection. Right: Mask of the detected objects. Scale bar: 3 $\mu \mathrm{m}$.

Table 1. Useful Drosophila lines for detection of wild-type or pathological fluorescent SG proteins. 